\title{
Freezing in the hierarchy problem
}

\author{
Timothy Cohen, ${ }^{1}$ Raffaele Tito D'Agnolo, ${ }^{2}$ and Matthew Low ${ }^{3}$ \\ ${ }^{1}$ Institute of Theoretical Science, University of Oregon, Eugene, Oregon 97403, USA \\ ${ }^{2}$ Theory Group, SLAC National Accelerator Laboratory, Menlo Park, California 94025, USA \\ ${ }^{3}$ School of Natural Sciences, Institute for Advanced Study, Princeton, New Jersey 08540, USA
}

(Received 11 September 2018; published 25 February 2019)

\begin{abstract}
Models with a tiny coupling $\lambda$ between the dark matter and the standard model, $\lambda \sim v / M_{\mathrm{Pl}} \sim 10^{-16}$, can yield the measured relic abundance through the thermal process known as freeze-in. We propose to interpret this small number in the context of perturbative large $N$ theories, where couplings are suppressed by inverse powers of $N$. Then $N \sim M_{\mathrm{Pl}}^{2} / v^{2}$ gives the observed relic density. Additionally, the ultimate cutoff of the standard model is reduced to $\sim 4 \pi M_{\mathrm{Pl}} / \sqrt{N} \sim 4 \pi v$, thereby solving the electroweak hierarchy problem. These theories predict a direct relation between the standard model cutoff and the dark matter mass, linking the spectacular collider phenomenology associated with the low gravitational scale to the cosmological signatures of the dark sector. The dark matter mass can lie in the range from hundreds of keV to hundreds of $\mathrm{GeV}$. Possible cosmological signals include washing out power for small scale structure, indirect detection signals from dark matter decays, and a continuous injection of electromagnetic and hadronic energy throughout the history of the Universe.
\end{abstract}

DOI: $10.1103 /$ PhysRevD.99.031702

\section{INTRODUCTIONS}

Dark matter (DM) accounts for $80 \%$ of the matter in our Universe, but its microscopic origin is still unknown. If the DM is coupled to the standard model (SM) thermal bath, a variety of mechanisms to produce the observed relic abundance are possible. The existence of a connection between the DM and the SM implies that measurements of these couplings today can teach us about the dynamics of the early Universe. One compelling thermal scenario is "freeze-in" [1], see [2] for a recent review. Initially, the SM is reheated and thermalizes, while the DM sector is not and therefore has a negligible energy density. As the Universe expands, the very small couplings to the SM mediate outof-equilibrium processes that generate a DM number density. The DM production ceases once the SM bath temperature $T$ becomes of order of the mass of the lightest SM particle that interacts with the DM, thereby freezing-in the relic density.

A key feature of freeze-in models is their very small coupling between the SM and DM. In this paper, we interpret this aspect in a new context, which leads us to find an interesting connection with the electroweak hierarchy problem. If the number $N$ of new states in the dark sector is large, but the theory is under perturbative control, the DM

Published by the American Physical Society under the terms of the Creative Commons Attribution 4.0 International license. Further distribution of this work must maintain attribution to the author(s) and the published article's title, journal citation, and DOI. Funded by SCOAP ${ }^{3}$. couplings to the SM must scale with an appropriate inverse power of $N$. Here we posit that this is the origin of the tiny couplings required for a viable model of freeze-in. We will show that $N \sim M_{\mathrm{Pl}}^{2} / v^{2}$ reproduces the observed relic density, where $v$ is the scale of electroweak symmetry breaking.

Theories with a huge number of new states are exciting for another reason. Gravity interacts with all the new degrees of freedom (d.o.f.). Therefore, graviton-graviton scattering at energy $E$ receives quantum corrections proportional to powers of $N E^{2} /\left(16 \pi^{2} M_{\mathrm{Pl}}^{2}\right)$ [3-5]. We expect the ultimate scale where gravity is modified to be reduced to $[6-10]$

$$
\Lambda_{\mathrm{UV}} \sim 4 \pi \frac{M_{\mathrm{Pl}}}{\sqrt{N}}
$$

If $N \sim M_{\mathrm{Pl}}^{2} / v^{2}$, this is a dramatic reduction in the size of the ultimate cutoff of the SM, and having $N$ dark sector states solves the hierarchy problem. In this paper we show that viable DM models exist with $N \sim 10^{15-35}$, implying $\Lambda_{\mathrm{UV}} \sim 1-10^{10} \mathrm{TeV}$.

If $\Lambda_{\mathrm{UV}}$ is within reach of the LHC or a future machine, then spectacular collider signatures associated with the gravitational sector would likely be discovered [9]. On the other hand, it is unsurprising that having $\Lambda_{\mathrm{UV}} \sim v$ comes at a price. In particular, new physics associated with gravity should appear at $\Lambda_{\mathrm{UV}}$, which can break baryon and/or lepton number, and can induce flavor-changing and/or $C P$-violating processes. One way to avoid the suite of associated constraints is to impose additional structure in 
the UV theory above $\Lambda_{\mathrm{UV}}$. Alternatively, it is perfectly viable to lift $\Lambda_{\mathrm{Uv}}$ above $v$, but at the expense of tuning the weak scale. For a given model, the freeze-in relic density calculation maps $N$ (and therefore $\Lambda_{\mathrm{UV}}$ ) onto a choice for the DM mass, as discussed in Secs. III and IV. This relation is one of the most appealing features of our framework since it implies that this class of theories is quite predictive: once we fix the large $N$ scaling of the couplings, the DM mass determines both the strength of its interactions with the SM and $\Lambda_{\mathrm{UV}}$.

Since at first blush introducing $N \sim 10^{\text {many }}$ new d.o.f. might seem extreme, it is worth briefly commenting on the connection to extra dimensional scenarios. One phenomenologically relevant example comes from introducing a mm-sized compactified extra dimension [11-14], which also modifies gravity at the $\mathrm{TeV}$ scale. In the $4 \mathrm{D}$ effective theory, this can be viewed as a consequence of a large number of Kaluza-Klein states, comparable to the number of species in our dark sector. In this sense, the equivalence of the large $N$ solution to the hierarchy problem can be made sharp. A similar emergence of large $N$ also occurs in calculable models of the AdS/CFT correspondence [15-18]. We emphasize that here we do not attempt to explain the origin of the $N$ dark sector states using an explicit extra dimensional construction, but making this connection precise, and understanding what theoretical restrictions it imposes, would be an interesting task for future work.

The rest of the paper is organized as follows. In Sec. II, we write down two example models and discuss their large $N$ scalings. Then in Sec. III, we present the calculation of the freeze-in abundance, and emphasize the modifications due to having $N$ species. Our numerical results and a discussion of the related phenomenology are given in Sec. IV, followed by a brief outlook.

\section{MODELS}

In this section, we introduce two example models that realize the goals set forth above. We also discuss the requirements imposed by perturbativity on their couplings. For context, in [19], 't Hooft demonstrated that in an $\mathrm{U}(N)$ gauge theory with gauge coupling $g$, holding the combination $g^{2} N$ fixed while taking the limit $N \rightarrow \infty$ maintains the validity of the perturbative expansion. This leads to a natural division into planar diagrams, which scale with powers of $g^{2} N$, and non-planar diagrams which are suppressed by powers of $1 / N$ with respect to the planar graphs. The perturbative expansion of the theory is reorganized in terms of the small parameter $1 / N$ [19].

We note that the relation between the topology of diagrams and the $1 / N$ expansion is a feature that we do not find in our simple models. This is due to the absence of fields that are the analog of the gluons in [19]. For both models presented here, we derive the minimal $N$ scaling of the coupling needed to maintain perturbativity. While what follows maintains the same spirit, our arguments differ from 't Hooft's approach in the details.

Scalar model: Our first example has $N$ real scalar DM candidates $\phi_{\alpha}$ coupled to the SM via the Higgs portal

$$
\mathcal{L} \supset-\lambda_{\phi}|H|^{2} \sum_{\alpha=1}^{N} \phi_{\alpha}^{2},
$$

whose stability is maintained by enforcing a $\mathbb{Z}_{2}$ symmetry: $\phi_{\alpha} \rightarrow-\phi_{\alpha}$. In the parameter space of interest, $m_{\phi_{\alpha}}<m_{h} / 2$, and the DM abundance is set by the decays $h \rightarrow \phi_{\alpha} \phi_{\alpha}$ after the electroweak phase transition. As discussed in Sec. IV, the $\phi_{\alpha}$ do not introduce $N$ hierarchy problems, given the tiny size of the coupling $\lambda_{\phi}$.

Every diagram constructed from the interaction in Eq. (2) contains a factor $r$, which scales as

$$
r \sim \lambda_{\phi}^{V} N^{L_{\phi}},
$$

where $V$ is the total number of vertices, and $L_{\phi}$ is the number of closed $\phi_{\alpha}$ loops. To make the structure of the perturbative expansion more manifest we would like to express $L_{\phi}$ in terms of $V$.

For any diagram, if we remove all external Higgs lines and internal Higgs propagators, we are left with $n$ disconnected subdiagrams (where $n \geq 1$ ) consisting only of $\phi_{\alpha}$ lines. We can then write

$$
L_{\phi}=\sum_{i=1}^{n} L_{\phi, i}=\sum_{i=1}^{n}\left(V_{i}-P_{i}+1\right)=V-P+n,
$$

where $V_{i}$ and $P_{i}$ are the number of vertices and edges respectively in the $i$ th subdiagram, $V \equiv \sum_{i=1}^{n} V_{i}$ and $P \equiv \sum_{i=1}^{n} P_{i}$. Then Eq. (3) becomes

$$
r \sim\left(\lambda_{\phi} N\right)^{V} N^{-P+n} .
$$

Since every subdiagram has at least one edge, $P \geq n$ and perturbativity is maintained by imposing $\lambda_{\phi} \lesssim 1 / N$. In particular, the leading diagrams scale as $\left(\lambda_{\phi} N\right)^{V}$ and consist of a single vertex per $\phi_{\alpha}$ loop $^{1}$

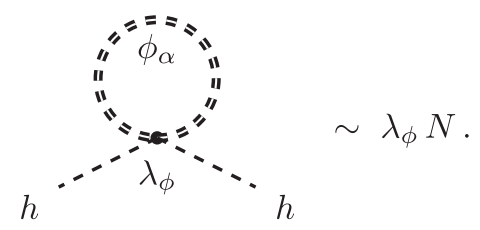

Since we consider also dark matter candidates with $m_{\phi} \ll$ $m_{h}$ this scaling of the coupling is not sufficient to insure

\footnotetext{
${ }^{1}$ In this theory, the leading diagrams renormalize the Higgs mass and the cosmological constant. If one is willing to fine-tune the Higgs mass, then perturbativity can be maintained with a weaker scaling $\lambda_{\phi} \sim 1 / \sqrt{N}$.
} 
that the masses of the new scalars are technically natural. This requirement introduces another constraint on the parameter space of the model that is discussed in Sec. IV.

Fermion model: Our second example introduces a fermionic DM candidate $\psi_{\alpha}$, that couples to the SM through the neutrino portal

$$
\mathcal{L} \supset-y_{\psi} L_{e} H \sum_{\alpha=1}^{N} \psi_{\alpha},
$$

where $L_{e}$ is the SM lepton doublet containing the electron. We have chosen to couple only to the electron doublet mainly for simplicity. Given the relevant size of $y_{\psi}$ discussed below, generalizing this coupling would have a minimal impact on the DM phenomenology, and as such we leave exploring this larger parameter space to future work. The small size of $y_{\psi}$ also insures that the $\psi_{\alpha}$ are stable on cosmological timescales, as discussed in more detail in Sec. IV.

In this model, ${ }^{2}$ each diagram contains a factor

$$
r \sim y_{\psi}^{V} N^{I_{\psi}}
$$

where $V$ is the total number of vertices, as above, and $I_{\psi}$ is the number of internal $\psi_{\alpha}$ propagators. As there is only one $\psi_{\alpha}$ line coming out of each vertex, we can write

$$
V=2 I_{\psi}+E_{\psi},
$$

where $E_{\psi}$ are external $\psi_{\alpha}$ lines. Then

$$
r \sim\left(y_{\psi}^{2} N\right)^{V / 2} N^{-E_{\psi} / 2} .
$$

The 't Hooft coupling is $y_{\psi}^{2} N$ and perturbativity is maintained by requiring $y_{\psi} \lesssim 1 / \sqrt{N}$. The leading diagrams scale as $y_{\psi}^{2} N$ and have no external $\psi_{\alpha}$ lines

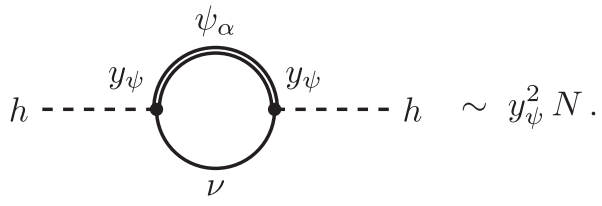

As we will show in the next two sections, both the scalar and fermion model can realize the measured relic abundance via freeze-in, while satisfying all the relevant phenomenological constraints.

\footnotetext{
${ }^{2}$ In this model, there is also a potential issue with maintaining unitarity at tree-level, for example in the process $h \nu \rightarrow h \nu$. The same 't Hooft scaling we derive at loop-level, also maintains the finiteness of the tree processes in the large $N$ limit.
}

\section{RELIC DENSITY}

Now that we have discussed the interplay between the requirement of perturbativity and the large $N$ scalings of the couplings, we turn to the calculation of the thermal freeze-in relic abundance. The goal of this section is to review the mechanism [1] with an emphasis on dark sectors with a large number of new species. We begin with a discussion of the parametrics, which are then derived using the full Boltzmann equations.

The freeze-in abundance of a single species due to the decay of an SM particle with mass $m_{\mathrm{SM}}$ is

$$
Y_{N=1} \equiv \frac{n_{N=1}}{s} \sim \lambda^{2} \frac{M_{\mathrm{Pl}}}{m_{\mathrm{SM}}},
$$

where $\lambda$ is the coupling between the SM and the new particle. The observed relic abundance is reproduced when $Y \sim v^{2} /\left(M_{\mathrm{P} 1} m_{\mathrm{DM}}\right)$, implying that

$$
\lambda \sim \frac{v}{M_{\mathrm{Pl}}} \sqrt{\frac{m_{\mathrm{SM}}}{m_{\mathrm{DM}}}} .
$$

In our scalar scenario defined in Eq. (2), we have shown that the coupling should scale at most as $1 / N$. Even when this scaling is fixed, there is a range of possibilities for the many particle abundance $Y_{N}$. If the couplings are diagonal, then only the channels $\mathrm{SM} \rightarrow \mathrm{DM}_{\alpha} \mathrm{DM}_{\alpha}$ are active so that the sum over the particles in the final state gives $Y_{N}=N Y_{N=1}$, and Eq. (13) becomes

$$
N \sim \frac{M_{\mathrm{Pl}}^{2}}{v^{2}} \frac{m_{\mathrm{DM}}}{m_{\mathrm{SM}}} \quad \text { (scalar model). }
$$

To simplify the parametrics, we have assumed that all the dark sector particles have the same mass and coupling to the SM.

On the other hand, if the couplings are all active, then $\mathrm{SM} \rightarrow \mathrm{DM}_{\alpha} \mathrm{DM}_{\beta}$ with $\alpha \neq \beta$ are allowed, giving $N(N+1) / 2$ decay channels. Note however that this possibility only makes sense for scenarios where the scalar masses are not all equal or in the presence of additional interactions. Putting it all together, again in the simple limit where all the couplings and masses are the same, we have

$$
\begin{aligned}
Y_{N=1} & \leq Y_{N} \lesssim N^{2} Y_{N=1} \\
& \Rightarrow \frac{1}{N} \frac{v}{M_{\mathrm{Pl}}} \sqrt{\frac{m_{\mathrm{SM}}}{m_{\mathrm{DM}}}} \lesssim \lambda_{\phi} \lesssim \frac{v}{M_{\mathrm{Pl}}} \sqrt{\frac{m_{\mathrm{SM}}}{m_{\mathrm{DM}}}} .
\end{aligned}
$$

We see that in theories where the off-diagonal decay channels are active, we have to suppress the coupling beyond the 't Hooft scaling in order to reproduce the observed relic density.

In our fermionic scenario defined in Eq. (7), DM candidates couple to the SM via a seesaw type mixing 

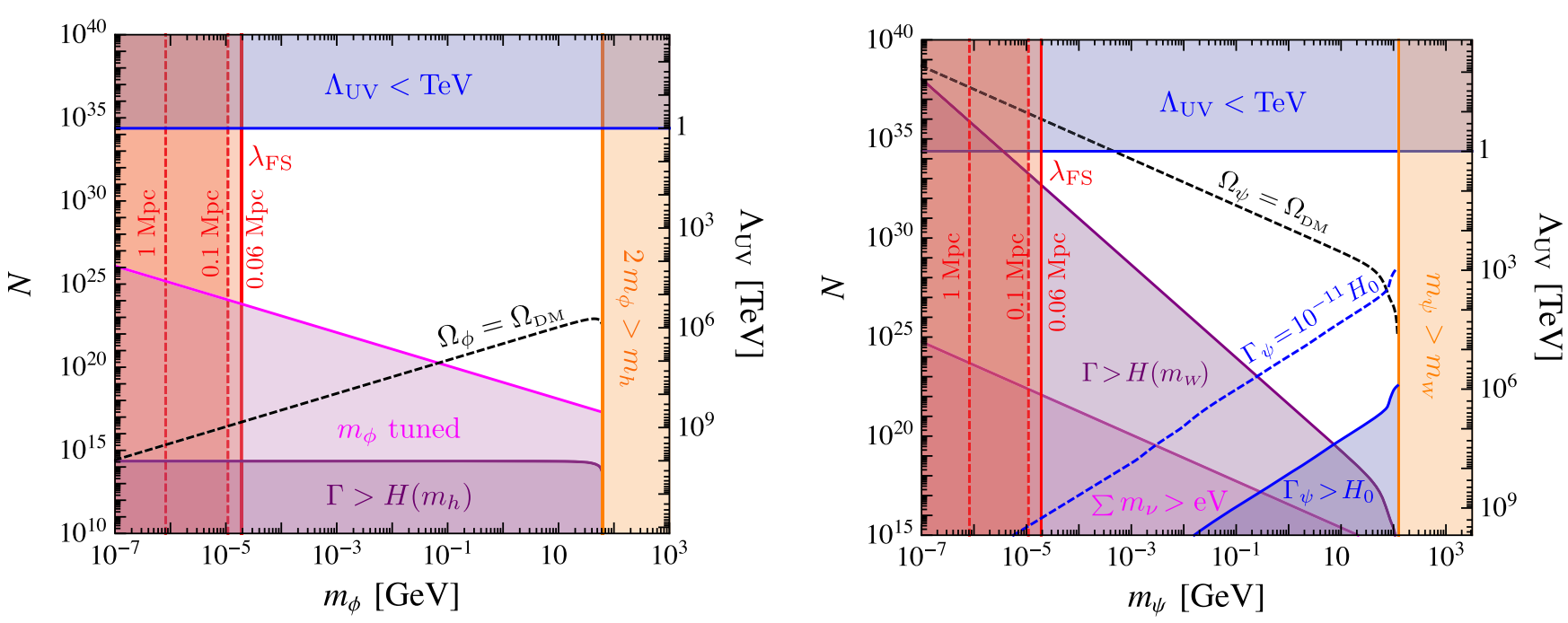

FIG. 1. Left: Phenomenology of the scalar DM model defined by Eq. (2). The DM is produced by $h \rightarrow \phi_{\alpha} \phi_{\alpha}$ decays. Right: Phenomenology of the fermion DM model defined by Eq. (7). The DM is dominantly produced by $W^{ \pm} \rightarrow e^{ \pm} \psi_{\alpha}$ decays. Note that in the parameter space below the relic density line $\Omega=\Omega_{\mathrm{DM}}$ the DM overcloses the Universe if a standard cosmological history is assumed. The figure is discussed in detail in Sec. IV.

with neutrinos. In this case their decay width is suppressed by an extra factor $v^{2} / m_{\mathrm{DM}}^{2}$ and a simple possibility is to have couplings $\sim 1 / N$ and $N$ decay channels. ${ }^{3}$ This gives

$$
N \sim \frac{M_{\mathrm{Pl}}^{2}}{m_{\mathrm{SM}} m_{\mathrm{DM}}} \quad(\text { fermion model })
$$

We will see in Sec. IV that even though they mix with the neutrinos, these DM candidates can easily be stable on cosmological timescales.

In the Supplemental Material [20], we show how these scaling result from the Boltzmann equations. If we take all the particles in the hidden sector to have the same mass (or assume that they all eventually decay to the lightest member of their sector), the relic density today is approximately given by

$$
\Omega h^{2} \simeq \frac{1.1 \times 10^{27} g_{\mathrm{SM}}}{g_{*}^{3 / 2}\left(m_{\mathrm{SM}}\right)} \frac{m_{\mathrm{DM}}}{m_{\mathrm{SM}}^{2}} \sum_{\alpha=1}^{N} \sum_{\beta=1}^{N} \Gamma_{\alpha \beta},
$$

in good agreement with a full numerical calculation. Here $g_{*} \simeq g_{* S}$ counts the effective number of d.o.f. in the SM thermal bath. This derivation shows that our multi-particle yield, $Y_{N}$, is proportional to the sum of $\Gamma_{\alpha \beta}=\Gamma(\mathrm{SM} \rightarrow$ $\mathrm{DM}_{\alpha} \mathrm{DM}_{\beta}$ ) over all the active channels.

\footnotetext{
${ }^{3}$ This choice anticipates the phenomenological constraints discussed in the next section when $y_{\psi} \sim 1 / \sqrt{N}$ and there are $N$ active decay channels.
}

\section{PHENOMENOLOGY}

In this section, we present the experimentally viable parameter space for our scalar and fermion models along with the associated signatures. We also make quantitative the relation between the DM mass and the gravitational cutoff. First, we assume that all particles have identical masses, which allows us to clearly illustrate the relevant constraints and the correlation between the DM mass and $\Lambda_{\mathrm{UV}}$. We then discuss how the phenomenology is modified in the case that the dark sector masses follow a non-trivial distribution in the supplemental material.

\section{A. Equal masses}

In our first example model, the DM is composed of $N$ scalar particles with masses $m_{\phi} \lesssim m_{h} / 2$ that couple to the SM through the Higgs portal as in Eq. (2). For simplicity, we enforce that the DM is stable, which implies that none of the $\phi_{\alpha}$ states obtain a vacuum expectation value. Then the DM relic density freezes-in after the electroweak phase transition from two-body decays of the Higgs, $h \rightarrow \phi_{\alpha} \phi_{\alpha}$. The abundance produced during the electroweak symmetric phase from the process $H H^{\dagger} \rightarrow \phi_{\alpha} \phi_{\alpha}$ is negligible. If only diagonal decays are active, then saturating the 't Hooft scaling of the coupling, $\lambda_{\phi}=1 / N$, yields the observed relic density along the black curve in the left panel of Fig. 1. Furthermore, this figure shows that the parameter space for the scalar model has a lower bound on the cutoff, $\Lambda_{\mathrm{UV}} \gtrsim 10^{6} \mathrm{TeV}$. In this model, $\Lambda_{\mathrm{UV}} \sim m_{\phi}^{-1 / 2}$ as determined by Eq. (14), such that the cutoff is lowest ( $N$ largest) for the largest kinematically allowed DM mass, in this case set by $m_{h}$. 
In our second example model, the DM is made up of $N$ fermions with masses $m_{\psi} \lesssim m_{W}$. They couple to the SM via the neutrino portal in Eq. (7), i.e., the same couplings as right-handed neutrinos. As discussed above, perturbativity requires $y_{\psi} \lesssim 1 / \sqrt{N}$ and the process $W^{ \pm} \rightarrow \psi_{i} e^{ \pm}$dominantly determines the freeze-in abundance. Saturating the perturbativity bound on $y_{\psi}$ can never yield the measured relic density without violating phenomenological constraints. This is straightforward to see by taking Eq. (17) and evaluating it at $T_{\mathrm{FI}} \simeq m_{W}$, which yields $\Omega_{\psi} h^{2} \simeq$ $3 \times 10^{24} m_{\psi} / m_{W}$, implying $m_{\psi} \simeq 10^{-15} \mathrm{eV}$. As discussed below, such a light mass is ruled out by measurements of structure formation.

To find phenomenologically viable parameter space, we can either reduce the number of decay channels, or note that if $y_{\psi} \ll N^{-0.5}$ it is easy to reproduce the correct relic density-for concreteness, results are shown in Fig. 1 for the (arbitrary) choice $y_{\psi}=N^{-0.9}$. We see that the cutoff can easily be brought down to the weak scale or even below with the fermion model. This is shown in the right panel of Fig. 1. In this case, the mixing with neutrinos impacts the relation between $N$ and the DM mass [see Eq. (16)], implying a scaling $\Lambda_{\mathrm{UV}} \sim m_{\psi}^{1 / 2}$.

Next we turn to the experimental bounds of our parameter space, most of which apply to both models. As $N$ becomes large enough, the gravitational cutoff passes below a $\mathrm{TeV}$, which is in conflict with multiple observations. At small $N$, the coupling between the dark sector and the SM becomes too large, and we are no longer in the freeze-in regime. In the plots, this is denoted by the shaded region where $\Gamma>H\left(m_{\mathrm{SM}}\right)$, i.e., the decay rate that populates the dark sector enters equilibrium at the freeze-in temperature. In the fermion model, another slice of small $N$ parameter space is excluded if $\Gamma_{\psi}>H_{0}$, the requirement of stability on timescales comparable to the age of the Universe.

The DM mass is also bounded from above and from below in both models. As $m_{\mathrm{DM}}$ approaches the weak scale, the SM to dark sector decays become kinematically forbidden. ${ }^{4}$ For simplicity, we derive this kinematic bound in the zero temperature limit, since at $T_{\mathrm{FI}} \simeq m_{\mathrm{SM}}$ thermal corrections are negligible for our purposes.

In both models, one lower bound on the mass follows from considering structure formation. In order to derive a constraint, we need to estimate the free-streaming length $\lambda_{\mathrm{FS}}$. In Fig. 1, we show the regions where $\lambda_{\mathrm{FS}} \gtrsim 0.06 \mathrm{Mpc}, 0.1 \mathrm{Mpc}$, and $1 \mathrm{Mpc}$. Currently, $m_{\mathrm{DM}} \lesssim$ $0.01 \mathrm{MeV}$ is in tension with observations from Lyman- $\alpha$ forest data [21,22].

It might seem that the tiny coupling between the dark sector and the SM precludes any hope of a positive signal

\footnotetext{
${ }^{4}$ We can still populate the dark sector through inverse decays, but we do not consider this regime here.
}

other than the possible suppression of the small scale power spectrum. However, since all of DM is unstable in the fermion model there are strong constraints from indirect detection. For masses above $10 \mathrm{GeV}$, the bounds from a dedicated analysis of Fermi gamma rays [23] are relevant, while below a combination of gamma-ray and x-ray data are more important [24]. While a reinterpretation for the decays $\psi_{\alpha} \rightarrow W^{(*)} \ell, Z^{(*)} \nu, h^{(*)} \nu$ is left for future work, we show the line through our parameter space in the right panel of Fig. 1 where $\Gamma_{\psi} \simeq 10^{-28} / \mathrm{s}$. Above this line DM is more long-lived and the parameter space is unconstrained, while a rough reinterpretation of [23] suggests that in our model everything below this line, $m_{\psi} \gtrsim 70 \mathrm{GeV}$, is excluded. That these models could be discovered using indirect detection is remarkable in view of the smallness of the coupling between DM and the $\mathrm{SM} 1 / N \sim v^{2} / M_{\mathrm{Pl}}^{2} \sim 10^{-33}$, and leaves open the possibility of exploring more of our parameter space with future indirect detection experiments, see, e.g., [25] for a discussion.

This completes the picture of the possible signals and the bounds from experiment. However, there is one important theoretical consideration to discuss for the scalar model. When the coupling $\lambda_{\phi}$ becomes sufficiently large, the quartic $\phi-H$ coupling in Eq. (2) introduces a quadratic correction to the $\phi_{\alpha}$ mass at one-loop. The area shaded in magenta in the left panel of Fig. 1 indicates a naive tuning bound where $\lambda_{\phi} \Lambda_{\mathrm{UV}}^{2} /\left(16 \pi^{2}\right) \gtrsim m_{\phi}^{2}$. Also, their tree-level contribution to the sum of the SM neutrino masses is well below an $\mathrm{eV}$ in most of the parameter space, as shown in the right panel of Fig. 1.

\section{OUTLOOK}

In this paper, we have made the connection between the large $N$ solution to the electroweak hierarchy problem and the freeze-in mechanism to generate the relic abundance of DM. We provided two simple models both of which introduce $N$ dark sector states, and an interaction with the SM whose size is determined by a 't Hooft coupling. We showed that it is indeed possible to achieve a phenomenologically viable scenario with smoking-gun signatures.

In the most interesting parameter space where the full hierarchy problem is solved, the ultraviolet cutoff is within reach of current and/or future colliders. Therefore, it should be possible to produce the $N$ states constituting dark matter through their gravitational interactions. Furthermore, in the case of the fermionic model, we can also directly access the freeze-in coupling of dark matter through indirect detection, by measuring its lifetime. This is a rather unique example in which dark matter interacts only gravitationally or through extremely feeble couplings, but we can still produce it directly and probe its freeze-in interactions.

The literature does contain ideas with a similar spirit, e.g., a class of large $N$ DM models [10], dynamical DM 
[26-28], and $N$ naturalness [29]. In all these cases, the DM can also be composed by a large number of species that could in principle contribute to lowering the gravitational cutoff of the theory. The framework presented here fits nicely within this spectrum of ideas. Our models are radical in their simplicity - the hierarchy problem and the nature of DM could be deeply linked, with observable consequences that could show up in future collider and indirect detection experiments.

\section{ACKNOWLEDGMENTS}

We would like to thank A. Berlin, S. A. R. Ellis, M. Lisanti, J. Maldacena, G. Marques-Tavares, R. Rattazzi, and L. Vecchi for useful discussions. T.C. is supported by the U.S. Department of Energy, under Grant No. DE-SC0011640. R. T. D. is supported by the U.S. Department of Energy under Grant No. DE-AC02-76SF00515. M. L. acknowledges support from the Institute for Advanced Study.
[1] L. J. Hall, K. Jedamzik, J. March-Russell, and S. M. West, Freeze-in production of FIMP dark matter, J. High Energy Phys. 03 (2010) 080.

[2] N. Bernal, M. Heikinheimo, T. Tenkanen, K. Tuominen, and V. Vaskonen, The dawn of FIMP dark matter: A review of models and constraints, Int. J. Mod. Phys. A 32, 1730023 (2017).

[3] D. C. Dunbar and P. S. Norridge, Calculation of graviton scattering amplitudes using string based methods, Nucl. Phys. B433, 181 (1995).

[4] X. Calmet and S. D. H. Hsu, TeV gravity in four dimensions?, Phys. Lett. B 663, 95 (2008).

[5] M. M. Anber and J.F. Donoghue, On the running of the gravitational constant, Phys. Rev. D 85, 104016 (2012).

[6] G. Dvali, Black holes and large $N$ species solution to the Hierarchy problem, Fortschr. Phys. 58, 528 (2010).

[7] G. Dvali and M. Redi, Black hole bound on the number of species and quantum gravity at LHC, Phys. Rev. D 77, 045027 (2008).

[8] G. Dvali and G. R. Farrar, Strong CP Problem with $10^{32}$ Standard Model Copies, Phys. Rev. Lett. 101, 011801 (2008).

[9] G. Dvali and M. Redi, Phenomenology of $10^{32}$ dark sectors, Phys. Rev. D 80, 055001 (2009).

[10] G. Dvali, I. Sawicki, and A. Vikman, Dark matter via many copies of the standard model, J. Cosmol. Astropart. Phys. 08 (2009) 009.

[11] N. Arkani-Hamed, S. Dimopoulos, and G. R. Dvali, The Hierarchy problem and new dimensions at a millimeter, Phys. Lett. B 429, 263 (1998).

[12] I. Antoniadis, N. Arkani-Hamed, S. Dimopoulos, and G. R. Dvali, New dimensions at a millimeter to a Fermi and superstrings at a TeV, Phys. Lett. B 436, 257 (1998).

[13] N. Arkani-Hamed, S. Dimopoulos, and G. R. Dvali, Phenomenology, astrophysics and cosmology of theories with submillimeter dimensions and $\mathrm{TeV}$ scale quantum gravity, Phys. Rev. D 59, 086004 (1999).

[14] G. F. Giudice, R. Rattazzi, and J. D. Wells, Quantum gravity and extra dimensions at high-energy colliders, Nucl. Phys. B544, 3 (1999).

[15] J. M. Maldacena, The large $N$ limit of superconformal field theories and supergravity, Int. J. Theor. Phys. 38, 1113 (1999); [Adv. Theor. Math. Phys. 2, 231 (1998)].
[16] E. Witten, Anti-de Sitter space and holography, Adv. Theor. Math. Phys. 2, 253 (1998).

[17] S. S. Gubser, I. R. Klebanov, and A. M. Polyakov, Gauge theory correlators from noncritical string theory, Phys. Lett. B 428, 105 (1998).

[18] O. Aharony, S. S. Gubser, J. M. Maldacena, H. Ooguri, and Y. Oz, Large $N$ field theories, string theory and gravity, Phys. Rep. 323, 183 (2000).

[19] G. 't Hooft, A planar diagram theory for strong interactions, Nucl. Phys. B72, 461 (1974).

[20] Please see the Supplemental Material at http://link.aps.org/ supplemental/10.1103/PhysRevD.99.031702 for details of the Boltzmann equations for computing the freeze-in abundance, and a brief discussion of how non-trivial mass distributions impact the physics.

[21] V. Iri et al., New constraints on the free-streaming of warm dark matter from intermediate and small scale Lyman- $\alpha$ forest data, Phys. Rev. D 96, 023522 (2017).

[22] M. Viel, G. D. Becker, J. S. Bolton, and M. G. Haehnelt, Warm dark matter as a solution to the small scale crisis: New constraints from high redshift Lyman- $\alpha$ forest data, Phys. Rev. D 88, 043502 (2013).

[23] T. Cohen, K. Murase, N. L. Rodd, B. R. Safdi, and Y. Soreq, $\gamma$-Ray Constraints on Decaying Dark Matter and Implications for IceCube, Phys. Rev. Lett. 119, 021102 (2017).

[24] R. Essig, E. Kuflik, S. D. McDermott, T. Volansky, and K. M. Zurek, Constraining light dark matter with diffuse $\mathrm{X}$-ray and gamma-ray observations, J. High Energy Phys. 11 (2013) 193.

[25] J. M. Gaskins, A review of indirect searches for particle dark matter, Contemp. Phys. 57, 496 (2016).

[26] K. R. Dienes and B. Thomas, Dynamical dark matter: I. Theoretical overview, Phys. Rev. D 85, 083523 (2012).

[27] K. R. Dienes and B. Thomas, Dynamical dark matter: II. An explicit model, Phys. Rev. D 85, 083524 (2012).

[28] K. R. Dienes, J. Fennick, J. Kumar, and B. Thomas, Randomness in the dark sector: Emergent mass spectra and dynamical dark matter ensembles, Phys. Rev. D 93, 083506 (2016).

[29] N. Arkani-Hamed, T. Cohen, R. T. D’Agnolo, A. Hook, H. D. Kim, and D. Pinner, Solving the Hierarchy Problem at Reheating with a Large Number of Degrees of Freedom, Phys. Rev. Lett. 117, 251801 (2016). 